\title{
Radiation from a Millimeter-Wave Rectangular Waveguide Slot Array Antenna Enclosed by a Von Karman Radome
}

\author{
Jihyung $\mathrm{Kim}^{1} \cdot$ Sung Chan Song ${ }^{1} \cdot$ Hokeun Shin ${ }^{2} \cdot$ Yong Bae Park ${ }^{2 *}$
}

\begin{abstract}
In this paper, electromagnetic radiation from a slot array antenna enclosed by a Von Karman radome is analyzed by using the ray tracing method and Huygens's principle. We consider the rectangular slot array antenna and the Von Karman radome. The radiation patterns are calculated by using the surface currents of the radome to illustrate the electromagnetic behaviors of the radome-enclosed waveguide slot array antenna.
\end{abstract}

Key Words: Ray Tracing Technique, Slot Array Antenna, Von Karman Radome.

\section{INTRODUCTION}

Radomes are widely used to protect antennas from their physical environment. As they distort the transmitted and received radar signals, they require electromagnetic analysis. Extensive studies have been conducted on the electromagnetic characteristics of radomes [1-14]. Ray tracing and physical optics (PO) method were suggested to analyze radomes [1]. Curved radomes enclosed in a single-element antenna were analyzed by using various methods [2-7]. The radiation pattern of a hemisphere radome fed from a circular aperture was calculated by using the dyadic Green's function technique/PO method [2] and the aperture integration-surface integration (AI-SI) [3]. The electromagnetic characteristics of the frequency selective surface tangent-ogive radome-enclosed antenna were analyzed using ray tracing and Huygens's principle [4]. The electromagnetic properties of the Von Karman radome-enclosed dipole antenna were analyzed by using the method of moments (MoM) [5] and coupled surface integral equation [6]. The radiation pattern of the Cassegrain monopulse antenna covered with a multilayered Von Karman radome was also analyzed by using the AI-SI [7]. The scattered fields of the Von Karman radome were calculated by using the MoM [8]. The radiation pattern of the conformal load-bearing antenna structure was calculated using the ray tracing-surface integration [9]. The boresight error (BSE) induced by a gimbal angle is analyzed by using the ray tracing technique [10]. Moreover, curved radomes enclosed by multiple sources were investigated using various analysis techniques [11-14]. The transmission through the radomeenclosed multiple sources was examined by using the multilevel fast multipole algorithm [11]. The hemisphere radome with a metallic cap was analyzed by using the time-domain finite integration theorem [12]. A tangent-ogive radome with a metallic cap was examined using the iterative PO (IPO)-boundary inte-

Manuscript received January 3, 2018 ; Revised February 19, 2018 ; Accepted April 8, 2018. (ID No. 20180103-001J)

${ }^{1}$ Hanwha Systems, Yongin, Korea.

${ }^{2}$ Department of Electrical and Computer Engineering, Ajou University, Suwon, Korea.

"Corresponding Author: Yong Bae Park (e-mail: yong@ajou.ac.kr)

This is an Open-Access article distributed under the terms of the Creative Commons Attribution Non-Commercial License (http://creativecommons.org/licenses/by-nc/4.0) which permits unrestricted non-commercial use, distribution, and reproduction in any medium, provided the original work is properly cited.

(c) Copyright The Korean Institute of Electromagnetic Engineering and Science. All Rights Reserved. 
gral-finite element method (FEM) [13]. The radiation properties of dipole arrays enclosed by the Von Karman radome were analyzed by using the IPO scheme [14]. However, the analysis of the hundreds of slot antennas enclosed by Von Karman radome based on an actual model was not presented. In this paper, we use the results of [15] for a waveguide array antenna with hundreds of rectangular slots and analyze the electromagnetic radiation from the antenna enclosed by a Von Karman radome based on the ray tracing technique and Huygens's principle. Our method is known to be numerically more efficient than the fullwave analysis or the IPO scheme. The rays from each slot are traced and the electromagnetic fields on the outer surface of the radomes are calculated by Huygens's principle. The radiation patterns are computed by using the surface currents on the outer surface of the radome to understand the electromagnetic characteristics of the radome-enclosed waveguide slot array.

\section{FIELD ANALYSIS}

The waveguide slot array antenna enclosed by a Von Karman radome is shown in Fig. 1. The waveguide array antenna has 112 elements [15]. The dielectric constant of the radome and tilt angle are $\varepsilon_{r}$ and $\alpha_{x}$. The shape of the Von Karman radome satisfies the following equations in the cylindrical coordinates.

$$
\begin{aligned}
& t=\arccos \left(1-\frac{2 z}{L_{i}}\right) \\
& \rho=\frac{R_{i}}{\sqrt{\pi}} \sqrt{t-\frac{\sin (2 t)}{2}}
\end{aligned}
$$

where $L_{i}, R_{i}$, and $t$ are the $i^{\text {th }}$ radome surface length, radius, and intervening variable, respectively. Fig. 2 illustrates the analysis procedure of the radome-enclosed waveguide slot array antenna based on the ray tracing and Huygens's principle.

The waveguide slot array antenna based on an actual model [15] has 112 elements and the electric fields of the slots are ob-

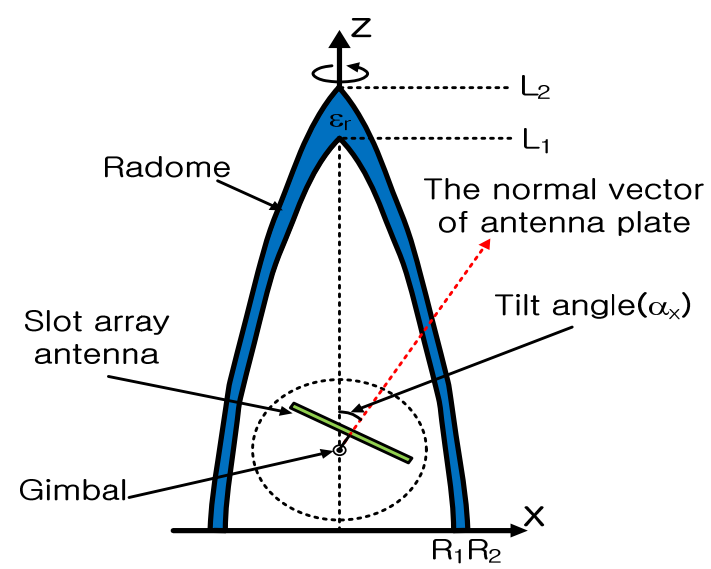

Fig. 1. Waveguide slot array antenna enclosed by a Von Karman radome.

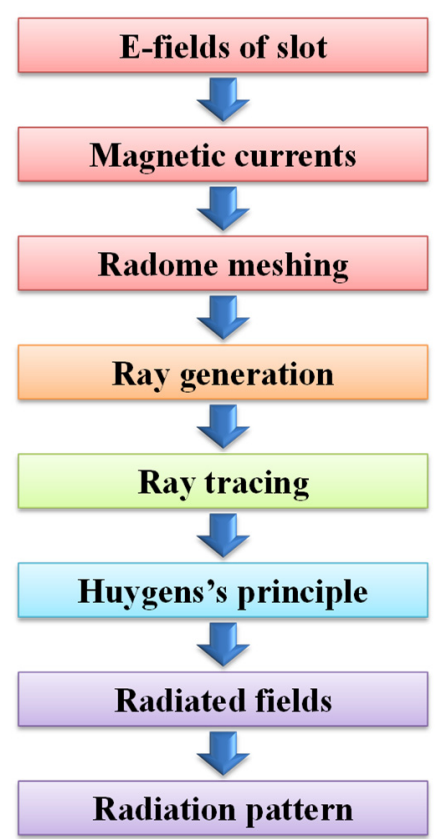

Fig. 2. Analysis procedure of the radome-enclosed waveguide slot array antenna.

tained by the simulation of the ANSYS High Frequency Structure Simulator (HFSS) based on the 3D FEM (Fig. 3). Details on how to design the antenna can be found in [15]. We assume that the point source is at the center of each slot. Using the surface equivalence theorem [16], the magnetic currents on the slot are given by

$$
\vec{M}=-2 \hat{n} \times \vec{E}_{t}
$$

where $\hat{n}$ is the normal vector of the slot and $\overrightarrow{E_{t}}$ is the tangential electric field of the slot. On the radome surface, the meshes are created in the vertical direction $(\hat{z})$ and in the azimuthal direction $(\hat{\phi})$ (Fig. 4). The radome surface is divided into $M$ (vertical direction) $\times N$ (azimuthal direction) meshes. We generate the rays from rectangular slots, the origins of which are the centers of the slots $\left(x_{0}, y_{0}, z_{0}\right)$ and the ray direction vector is $\overrightarrow{k_{l, j}}$.

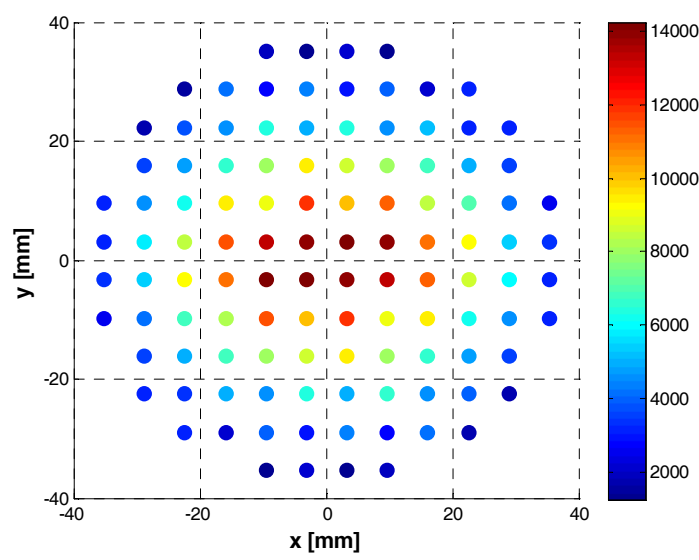

Fig. 3. Electric field of the rectangular slots. 


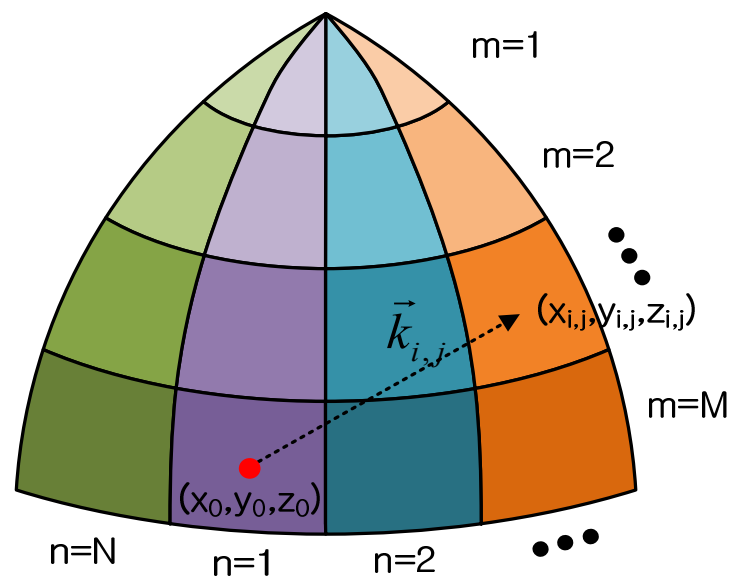

Fig. 4. Mesh and ray generation.

Note that $\left(x_{i j}, y_{i j}, z_{i j}\right)$ is the center of the mesh $(M=i$ and $N=j)$. To apply the ray tracing technique inside the radome, the ray path is calculated [4]. We find the intercept point of the ray incident on a ray radome surface based on the iterative method [1]. At the intercept point $\left(x_{j}, y_{i}, z_{i}\right)$, the normal vector $\left(\hat{n}_{v}\right)$ of the $i^{\text {th }}$ Von Karman radome surface can be expressed as

$$
\begin{gathered}
\hat{n}_{v}=\frac{x_{i}}{\sqrt{x_{i}^{2}+y_{i}^{2}}} \cdot \frac{t_{z}}{\sqrt{t_{\rho}^{2}+t_{z}^{2}}} \hat{x}+\frac{y_{i}}{\sqrt{x_{i}^{2}+y_{i}^{2}}} \cdot \frac{t_{z}}{\sqrt{t_{\rho}^{2}+t_{z}^{2}}} \hat{y}-\frac{t_{\rho}}{\sqrt{t_{\rho}^{2}+t_{z}^{2}}} \hat{z} \\
t_{\rho}=-\frac{R_{i}}{\sqrt{\pi}} \cdot \frac{(1-\cos (2 t))}{2 \sqrt{\frac{t-\sin (2 t)}{2}}} \\
t_{z}=\frac{L_{i}}{2 \sin (t)}
\end{gathered}
$$

where $t_{\rho}$ and $t_{z}$ are the horizontal and vertical components of the tangential vectors, respectively. At the intercept point, the incident fields are split by the perpendicular and parallel components. The reflected and transmitted waves are obtained by using the reflection and transmission coefficients of each polarization. On the outer radome surface, we enforce Huygens's principle to calculate the radiation pattern of the Von Karman radome-enclosed slot array antenna [4]. We use the far-field approximation [15] to calculate the radiated fields from each radome mesh as

$$
\begin{gathered}
E_{\theta(m, n)}=-\frac{j \beta e^{-j \beta r_{(m, n)}}}{4 \pi r_{(m, n)}}\left(L_{\phi(m, n)}+\eta N_{\theta_{(m, n)}}\right) \\
E_{\phi(m, n)}=\frac{j \beta e^{-j \beta r_{(m, n)}}}{4 \pi r_{(m, n)}}\left(L_{\theta(m, n)}-\eta N_{\phi(m, n)}\right) \\
H_{\theta(m, n)}=\frac{j \beta e^{-j \beta r_{(m, n)}}}{4 \pi r_{(m, n)}}\left(N_{\phi(m, n)}-\frac{L_{\theta(m, n)}}{\eta}\right) \\
H_{\phi(m, n)}=-\frac{j \beta e^{-j \beta r_{(m, n)}}}{4 \pi r_{(m, n)}}\left(N_{\theta(m, n)}+\frac{L_{\phi(m, n)}}{\eta}\right)
\end{gathered}
$$

$$
\begin{gathered}
N_{\theta(m, n)}=A_{(m, n)} \cdot\left(J_{x(m, n)} \cos \theta \cos \phi\right. \\
\left.+J_{y(m, n)} \cos \theta \sin \phi-J_{z(m, n)} \sin \theta\right) \\
N_{\phi(m, n)}=A_{(m, n)} \cdot\left(-J_{x(m, n)} \sin \phi+J_{y(m, n)} \cos \phi\right) \\
L_{\theta(m, n)}=A_{(m, n)} \cdot\left(M_{x(m, n)} \cos \theta \cos \phi\right. \\
\left.+M_{y(m, n)} \cos \theta \sin \phi-M_{z(m, n)} \sin \theta\right) \\
L_{\phi(m, n)}=A_{(m, n)} \cdot\left(-M_{x(m, n)} \sin \phi+M_{y(m, n)} \cos \phi\right)
\end{gathered}
$$

where $A_{(m, n)}$ is the surface area of each mesh as

$$
\begin{gathered}
A_{(m, n)}=\frac{2 \pi}{N} \cdot \int_{t_{\min }}^{t_{\max }} \frac{R_{2}}{\sqrt{\pi}} \sqrt{\left(t-\frac{\sin (2 t)}{2}\right)\left(\frac{R_{i}}{\sqrt{\pi}} \cdot \frac{1-\cos (2 t)}{-2 \sqrt{t-\sin (2 t) / 2}}+\left(-\frac{L_{2}}{2} \sin (t)\right)\right)} d t \\
t_{\max }=\arccos \left(1-\frac{2\left(L_{2}-z_{m}\right)}{L_{2}}\right) \\
t_{\text {min }}=\arccos \left(1-\frac{2\left(L_{2}-z_{m-1}\right)}{L_{2}}\right) \\
z_{m}=\frac{m \cdot L_{2}}{M}
\end{gathered}
$$

where, $L_{2}$ and $R_{2}$ are the length and the radius of the Von Karman radome, respectively. The radiated power can be obtained from the Poynting vector $(\vec{P})$ as

$$
\vec{P}=\frac{1}{2}\left(\operatorname{Re}\left(\vec{E} \times \vec{H}^{*}\right)\right)
$$

where $\vec{E}$ and $\vec{H}$ are the radiated electromagnetic fields at the observation points.

\section{NUMERICAL RESULTS}

To check the validity of our analysis, we consider the radome with a dielectric constant $\left(\varepsilon_{r}\right)$ of 1 , which means (that the array has no radome). Fig. 5 illustrates the radiation pattern of the Von Karman radome-enclosed waveguide slot array antenna designed for the Ka-band. We compare our calculated result with the radiation pattern of the slot array antenna. As the mesh number increases to $400 \times 400$, the results of the ray tracing tech-

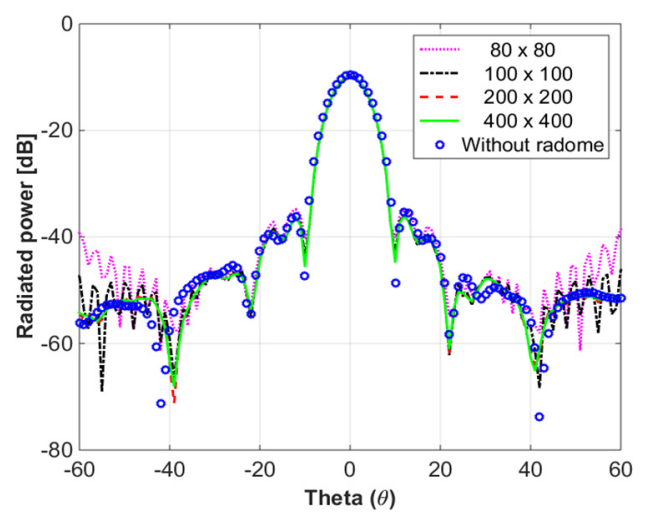

Fig. 5. Radiation pattern of the radome-enclosed waveguide slot array antenna $\left(\varepsilon_{r}=1\right)$. 
Table 1. Number of mesh and mesh size (outer surface)

\begin{tabular}{lc}
\hline Number of mesh $(\mathrm{M} \times \mathrm{N})$ & Mesh size $(\mathrm{z} \times \phi)$ \\
\hline $80 \times 80$ & $0.87 \lambda \times 0.061 \lambda-1.3 \lambda$ \\
$100 \times 100$ & $0.70 \lambda \times 0.041 \lambda-1.0 \lambda$ \\
$200 \times 200$ & $0.35 \lambda \times 0.012 \lambda-0.50 \lambda$ \\
$400 \times 400$ & $0.17 \lambda \times 0.0037 \lambda-0.25 \lambda$ \\
\hline
\end{tabular}

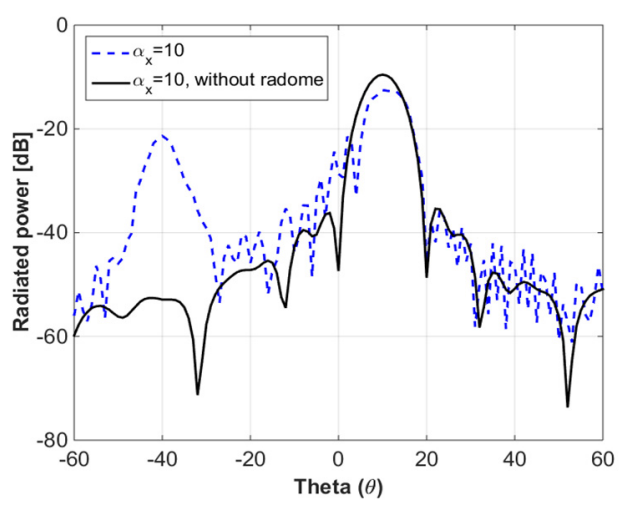

(a)

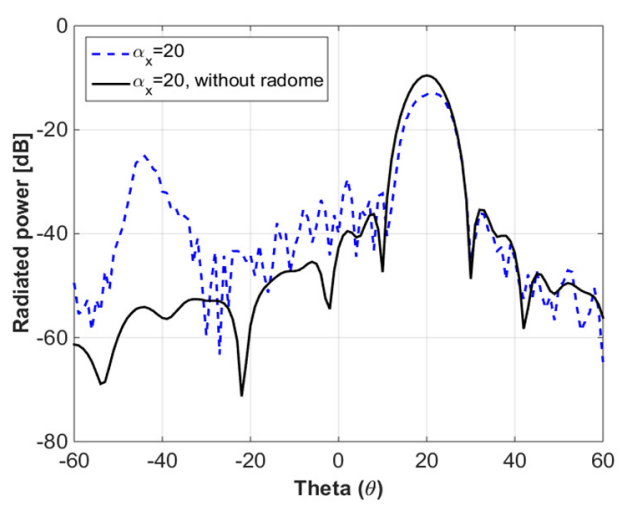

(b)

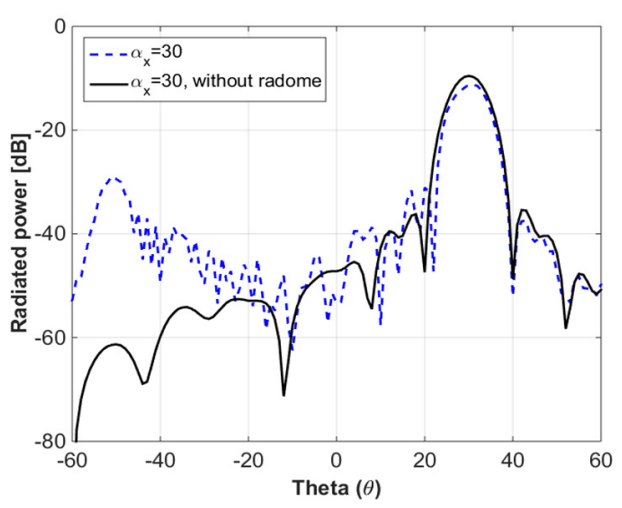

(c)

Fig. 6. Radiation pattern of the radome-enclosed waveguide slot array antenna. (a) $\alpha_{x}=10^{\circ}$, (b) $\alpha_{x}=20^{\circ}$, and (c) $\alpha_{x}=30^{\circ}$.

nique and the case of the slot antenna without a radome show a good agreement. Note that the mesh size should be less than $\lambda / 4$ (Table 1).

Fig. 6 shows the radiation pattern of the Von Karman radome-enclosed waveguide slot array antenna for the different gimbal tilt angles $\left(\alpha_{x}\right)$. The design parameters of the radome are presented in Table 2, and Table 3 shows the transmission loss of the radome. Two possibilities can account for the gain reduction. The transmission loss and the sidelobe level decrease as the tilt angle increases because of the enhancement of reflection loss

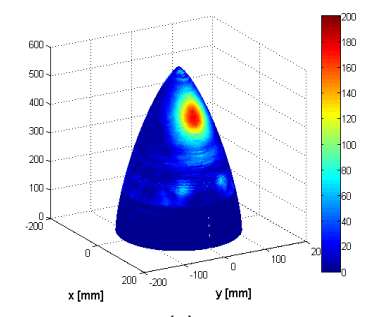

(a)

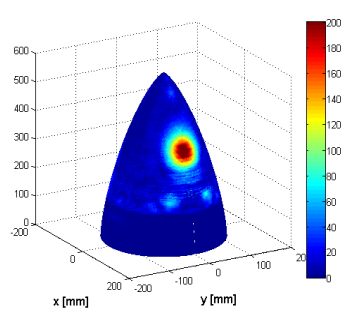

(c)

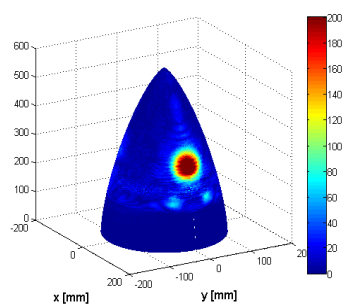

(e)

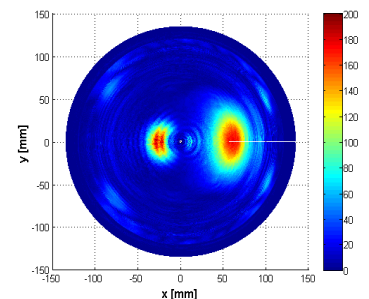

(b)

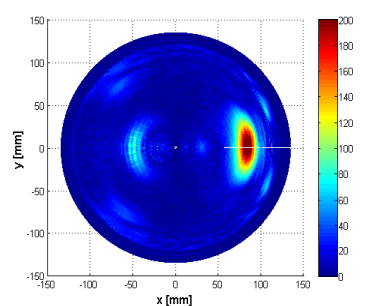

(d)

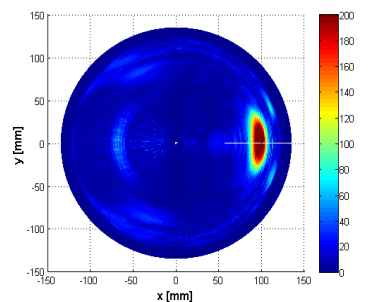

(f)
Fig. 7. Surface current of the radome. (a) $\alpha_{x}=10^{\circ}$ (side view), (b) $\alpha_{x}$ $=10^{\circ}$ (top view), (c) $\alpha_{x}=20^{\circ}$ (side view), (d) $\alpha_{x}=20^{\circ}$ (top view), (e) $\alpha_{x}=30^{\circ}$ (side view), (f) $\alpha_{x}=30^{\circ}$ (top view).

Table 2. Design parameter of the Von Karman radome in the case of Fig. 5

\begin{tabular}{lc}
\hline Parameter & Value \\
\hline Inner radius $\left(R_{1}\right)$ & $15.2 \lambda$ \\
Outer radius $\left(R_{2}\right)$ & $16.0 \lambda$ \\
Inner length $\left(L_{1}\right)$ & $66.9 \lambda$ \\
Outer length $\left(L_{2}\right)$ & $68.0 \lambda$ \\
$\varepsilon_{r}$ & 3.41 \\
\hline
\end{tabular}

Table 3. Transmission loss and the sidelobe level of the Von Karman radome-enclosed waveguide slot array antenna

\begin{tabular}{llll}
\hline & \multicolumn{3}{c}{ Gimbal tilt angle $\left(\alpha_{x}\right)$} \\
\cline { 2 - 4 } & $10^{\circ}$ & $20^{\circ}$ & $30^{\circ}$ \\
\hline Transmission loss $(\mathrm{dB})$ & -3.10 & -3.35 & -1.64 \\
Sidelobe level $(\mathrm{dB})$ & -9.29 & -16.55 & -20.44 \\
\hline
\end{tabular}


at smaller incident angles. Note that the image robes are observed at nearly $\theta=-40^{\circ}$ in Fig. 6 because of the internal reflection within the radome. Fig. 7 illustrates the surface currents of the radome for the different gimbal tilt angles $\left(\alpha_{x}\right)$. Large current distributions can be seen near the each tilt angle and the angle of the image robe.

\section{CONCLUSION}

We have analyzed the electromagnetic radiation from a slot array antenna enclosed by a Von Karman radome using the ray tracing technique and Huygens's principle. The radiation patterns of the radome-enclosed waveguide slot array antenna were calculated to illustrate the electromagnetic behaviors of the radomes. Our method is useful to estimate the electromagnetic characteristics, such as the radiation patterns and the BSE, of the radome-enclosed waveguide slot array antenna.

This work was supported by Hanwha Systems.

\section{REFERENCES}

[1] D. J. Kozakoff, Analysis of Radome-Enclosed Antennas. Boston, MA: Artech House, 1997.

[2] L. W. Li, M. S. Leong, X. Ma, and T. S. Yeo, "Analysis of a circular aperture antenna and its covered dielectric hemispherical radome shell over ground plane: near- and farzone patterns," Microwave and Optical Technology Letters, vol. 21, no. 4, pp. 238-243, 1999.

[3] J. Wang, G. Guo, H. Zheng, and E. Li, "Characteristic analysis of nose radome by aperture-integration and surfaceintegration method," in Proceedings of 2012 International Workshop on Microwave and Millimeter Wave Circuits and System Technology (MMWCST), Chengdu, China, 2012, pp. $1-4$.

[4] J. H. Kim, H. J. Chun, I. P. Hong, Y. J. Kim, and Y. B. Park, "Analysis of FSS radomes based on physical optics method and ray tracing technique," IEEE Antennas and Wireless Propagation Letters, vol. 13, pp. 868-871, 2014.

[5] E. Arvas, A. Rahhalarabi, E. Gundogan, and U. Pekel, "Electromagnetic transmission through a small radome of arbitrary shape," IEE Proceedings H (Microwaves, Antennas and Propagation), vol. 137, no. 6, pp. 401-405, 1990.
[6] M. He, X. Xu, B. Hu, and Y. Zheng, "Accurate analysis of arbitrarily shaped wire antenna-dielectric radome structures," IEEE Antennas and Wireless Propagation Letters, vol. 6, pp. 408-410, 2007.

[7] H. Meng, W. Dou, and K. Yin, "Analysis of antennaradome system at millimeter wave band," in Proceedings of 2008 Global Symposium on Millimeter Waves, Nanjing, China, 2008, pp. 380-383.

[8] H. Mustacoglu, J. R. Mautz, and E. Arvas, "Method of moments analysis of an axisymmetric chiral radome," in Proceedings of 2011 30th URSI General Assembly and Scientific Symposium, Istanbul, Turkey, 2011, pp. 1-4.

[9] P. Li, W. Y. Xu, L. W. Song, and Y. Y. Qiu, "A novel inversion method of manufacturing flaws in the packaging of conformal load-bearing antenna structure," International Journal of Antennas and Propagation, vol. 2015, article ID. 795323, 2015.

[10] P. L. Overfelt, "Superspheroids: a new family of radome shapes," IEEE Transactions on Antennas and Propagation, vol. 43, no. 2, pp. 215-220, 1995.

[11] C. C. Lu, "Dielectric radome analysis using multilevel fast multiple algorithm," in Proceedings of IEEE Antennas and Propagation Society International Symposium, Boston, MA, 2001, pp. 730-733.

[12] J. W. You, S. R. Tan, X. Y. Zhou, W. M. Yu, and T. J. Cui, "A new method to analyze broadband antennaradome interactions in time-domain," IEEE Transactions on Antennas and Propagation, vol. 62, no. 1, pp. 334-344, 2014.

[13] H. Meng and W. Dou, "Hybrid IPO-BI-FEM for the analysis of 2D large radome with complex structure," $M i$ crowave and Optical Technology Letters, vol. 51, no. 5, pp. 1348-1353, 2009.

[14] I. S. Koh, C. H. Park, and W. Sun, "Analysis of radiation characteristics of Ka-band von Karman radome based on IPO scheme," The Journal of Korean Institute of Electromagnetic Engineering and Science, vol. 22, no. 12, pp. 11481154, 2011.

[15] S. Trinh-Van, S. C. Song, S. H. Seo, and K. C. Hwang, "Waveguide slot array antenna with a hybrid-phase feed for grating lobe reduction," International Journal of Antennas and Propagation, vol. 2016, article ID. 4825924, 2016.

[16] C. A. Balanis, Advanced Engineering Electromagnetics. Hoboken, NJ: John Wiley \& Sons, 2012. 
Jihyung Kim

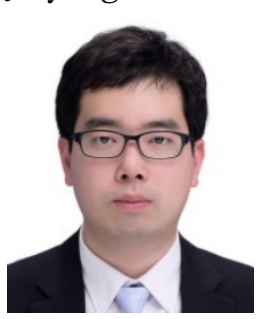

obtained his B.S. and M.S./Ph.D. degrees in electrical engineering from Ajou University, Suwon, Korea, in 2009 and 2016, respectively. Since 2016, he has been a researcher at Hanwha Systems. His research interests include the analysis of aperture array antennas and radomes.

\section{Sung Chan Song}

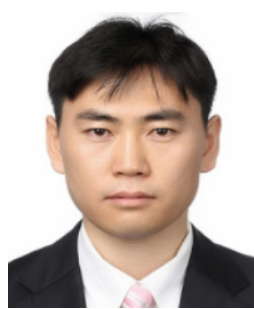

obtained his B.S. and M.S. degrees in avionics engineering from Korea Aerospace University, Goyang, Korea, in 2001 and 2003, respectively. From 2002 to 2015, he worked at Samsung Thales. Since 2015, he has been a researcher at Hanwha systems. His research interests include antennas, electromagnetic wave numerical analysis, and radar systems.

\section{Hokeun Shin}

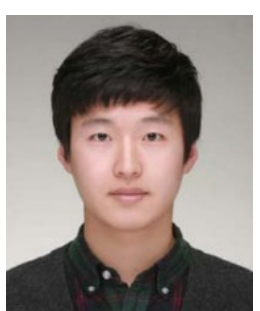

received his B.S. degree in electrical and computer engineering from Ajou University, Suwon, Korea, in 2015. He is currently undertaking his M.S. and Ph.D. course at the Department of Electrical and Computer Engineering, Ajou University, Suwon, Korea. His research interests include the analysis of radomes and radar cross section.

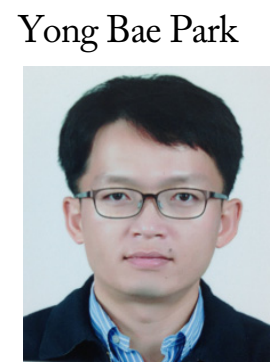

received his B.S. (summa cum laude), M.S., and $\mathrm{Ph} . \mathrm{D}$. degrees in electrical engineering from the Korea Advanced Institute of Science and Technology (KAIST), Daejeon, Korea, in 1998, 2000, and 2003, respectively. From 2003 to 2006, he was with the Korea Telecom Laboratory, Seoul, Korea. In 2006, he joined the School of Electrical and Computer Engineering, Ajou University, Suwon, Korea, where he is currently a professor. His research interests include electromagnetic field analysis and electromagnetic interference and compatibility. 